INRA Prod. Anim., 2011, 24 (2), 165-170

\title{
Sélection génétique et bien-être des poulets de chair et des reproducteurs
}

\author{
C. ARNOULD $1,2,3,4$, V. MICHEL ${ }^{5}$, E. LE BIHAN-DUVAL 6 \\ 1 INRA, UMR85 Physiologie de la Reproduction et des Comportements, F-37380 Nouzilly, France \\ ${ }^{2}$ CNRS, Physiologie de la Reproduction et des Comportements, F-37380 Nouzilly, France \\ 3 Université François Rabelais de Tours, F-37041 Tours France \\ ${ }^{4}$ IFCE, F-37380 Nouzilly, France \\ 5 Anses, F-22440 Ploufragan, France \\ ${ }^{6}$ INRA, UR83 Recherches Avicoles, F-37380 Nouzilly, France \\ Courriel : cecile.arnould@tours.inra.fr
}

La pression de sélection sur la croissance des poulets élevés pour la production de viande a été particulièrement forte au cours des dernières décennies. En parallèle, le questionnement des citoyens et du législateur sur leur niveau de bien-être s'est accentué. Le présent article expose les relations qui existent entre génétique et bien-être chez cette espèce, en s'appuyant sur les rapports produits en 2010 par l'Agence Européenne de Sécurité Alimentaire.

Depuis la seconde moitié du 20ème siècle, la sélection génétique du poulet de chair utilisé en filière standard a eu pour principal objectif un gain de productivité, grâce à l'augmentation de la vitesse de croissance et la diminution de l'indice de consommation. Ainsi, il faut actuellement 30 jours pour que ces poulets atteignent $1,5 \mathrm{~kg}$ alors qu'il en fallait 120 dans les années 50 . Simultanément l'indice de consommation est passé de 4,4 à 1,47. Le poulet étant abattu à poids fixe, l'augmentation des vitesses de croissance s'est accompagnée d'une diminution (de l'ordre d'un jour par an) de l'âge d'abattage et actuellement en Europe on peut considérer que les poulets de chair «standard» atteignent le poids de $2,5 \mathrm{~kg}$ en 42 jours en moyenne. La mise sur le marché d'animaux plus jeunes avec une viande plus tendre et au goût moins prononcé a aussi entraîné une diversification des filières de production avec, dès 1965, la création du Label Rouge attestant de la qualité supérieure du produit par rapport au standard (Beaumont et $a l$ 2004). Seules des souches à croissance lente permettant un abattage plus tardif (à un minimum de 81 jours) peuvent être utilisées pour la production du pou- let Label Rouge. Celle-ci impose également un cahier des charges strict sur les conditions d'élevage et notamment sur la densité d'élevage (limitée à 11 animaux par $\mathrm{m}^{2}$ ) et sur la taille restreinte des bâtiments d'élevage (pas plus de 4 bâtiments de $400 \mathrm{~m}^{2}$ par élevage). De telles règles ne s'imposent pas en filière standard où les poulets sont élevés dans des conditions plus intensives: fortes densités, groupes de plusieurs milliers d'individus (voir Arnould et Leterrier, 2007 pour plus de détails).

C'est dans ce contexte que la directive $2007 / 43 /$ CE sur la protection des poulets destinés à la production de viande a été adoptée en 2007 (JO L 182, 12.7.2007, 19-28). Cette directive, qui faisait suite au rapport de la Commission européenne, paru en 2000, sur le bien-être des poulets élevés pour la production de viande (SCAHAW 2000), stipule que la Commission doit soumettre au Parlement européen et au Conseil un rapport sur l'influence de la sélection génétique sur certaines déficiences identifiées qui conduisent à une dégradation du bien-être des poulets. En effet, le rapport publié en 2000 soulignait que de nombreuses caractéristiques métabo- liques et comportementales des poulets de chair avaient été modifiées par la sélection pour une croissance plus rapide et plus efficace et que la plupart des problèmes de bien-être observés chez ces animaux (troubles locomoteurs, ascites, syndrome de mort subite...) étaient directement liés à celle-ci. Le rapport concluait également qu'il existait des problèmes de bien-être liés à la façon dont les poulets reproducteurs étaient élevés, en particulier en ce qui concerne les restrictions alimentaires et les restrictions d'espace.

Dans ce contexte, la Commission européenne a demandé à l'European Food Safety Authority (EFSA) ${ }^{1}$ de produire deux avis scientifiques. Le premier concernait l'influence de la sélection génétique sur le bien-être des poulets de chair. Le second portait sur l'influence des conditions de logement et d'élevage sur le bien-être des reproducteurs de type parentaux et grandparentaux. En effet, les poulets standards élevés pour la production de viande (poulets de chair) correspondent le plus souvent à un croisement dit «4 voies» comprenant deux étapes de croisements entre des reproducteurs de

\footnotetext{
${ }^{1}$ L'EFSA (Agence Européenne de Sécurité Alimentaire) est une agence indépendante chargée d'émettre des avis et des conseils scientifiques afin d'aider la Commission européenne, le Parlement européen et les États membres de l’UE à arrêter des décisions en matière de gestion des risques, notamment dans le domaine du bien-être animal.
} 
Figure 1. Sélection et pyramide de multiplication d'une lignée commerciale de poulets de chair (d'après AHAW 2010a). Un petit nombre de compagnies produisent les différentes lignées utilisées à travers le monde $m$ : mâle, $f$ : femelle.

Année 0 Sélection pedigree

Lignées mâles

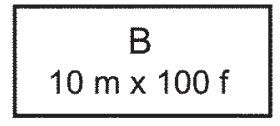
parentaux
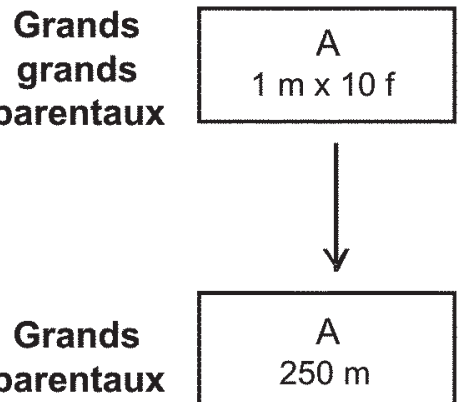
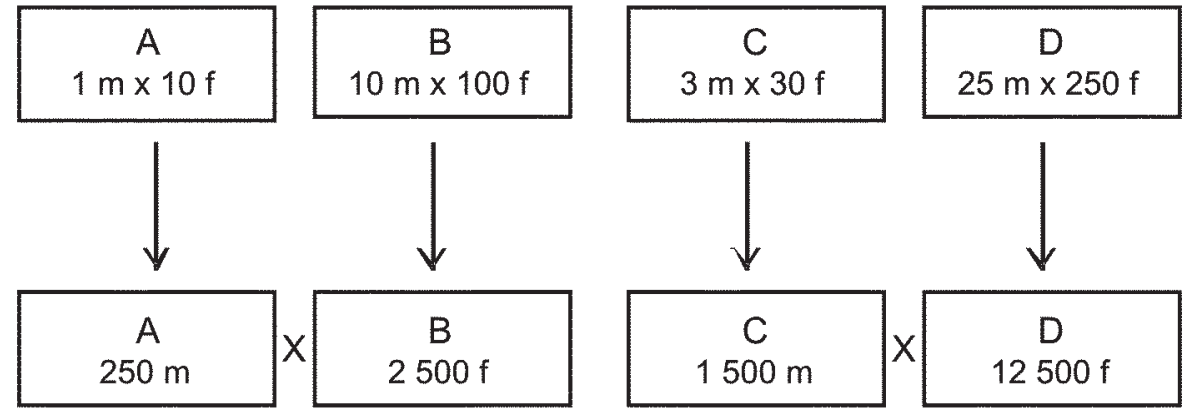
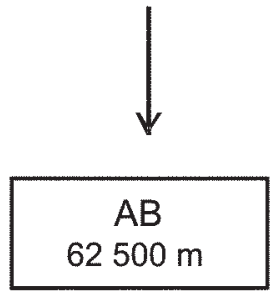

Lignées femelles
Année 3 Parentaux

\section{Années 4 à 5}

\section{Poulets de chair (croisements commerciaux)}

type grand-parentaux (issus des lignées pures en sélection) puis parentaux (figure 1). Grâce aux croisements successifs, ce schéma pyramidal assure la multiplication nécessaire à la production d'un grand nombre de poulets commerciaux tout en bénéficiant de la complémentarité entre les lignées dites mâles et femelles (soumises à des pressions de sélection différentes sur la croissance et la reproduction) ainsi que d'effets d'hétérosis favorables. Il assure, par ailleurs, une protection du matériel génétique. Les avis de l'EFSA ont été publiés en 2010 (AHAW 2010a et b) suite à une expertise scientifique qui s'est déroulée de juin 2009 à juillet 2010 .

Cette synthèse repose sur les principales conclusions et recommandations issues des deux avis de l'EFSA mentionnés ci-dessus. Par souci de simplification, seules les références qui n'apparaissent pas dans les avis de l'EFSA seront mentionnées dans cette synthèse. Cet article reviendra d'abord sur la présentation des conclusions et recommandations des deux rapports en lien avec l'influence de la sélection génétique sur le bien-être des poulets de chair et des reproducteurs, avant d'évoquer les domaines sur lesquels il reste des connaissances scientifiques à acquérir ou approfondir. Il se conclura par quelques priorités à prendre en compte pour faire évoluer les objectifs et pratiques de sélection dans un souci d'amélioration du bien-être des animaux.

\section{1 / Poulets de chair}

Cet article ne reviendra pas sur les différents problèmes de bien-être identifiés chez le poulet de chair qui ont déjà fait l'objet de synthèses (Arnould 2003, Arnould et Leterrier 2007). Elle se focalisera sur ceux en lien avec la sélection génétique.

La plupart des problèmes de bien-être chez les poulets de chair sont multifactoriels, c'est-à-dire qu'ils résultent à la fois de facteurs génétiques et de facteurs liés à l'environnement et à la gestion de l'élevage. Cependant, il est précisé au début du rapport que certains problèmes comme le syndrome de mort subite et l'ascite, les désordres squelettiques et la faible activité locomotrice, ou encore la faible tolérance au stress sont reconnus comme étant principalement liés à la sélection génétique pour une croissance rapide et une production plus efficace.

Même si la fréquence de certains problèmes de bien-être semble avoir diminué ces dernières années (comme les déformations de type varus-valgus au niveau des pattes ou l'ascite), il existe un manque de données de terrain sur leur évolution réelle. C'est pourquoi il est recommandé de mettre en place un système de surveillance de l'état des poulets, notamment par la mesure des problèmes locomoteurs, dermatites de contact, ascites, syndromes de mort subite, afin de suivre la fréquence de ces troubles dans les élevages commerciaux et de détecter l'apparition éventuelle de nouveaux problèmes.

\section{1 / Désordres musculo-squelet- tiques (troubles locomoteurs)}

Les désordres musculo-squelettiques sont à l'origine de sérieuses préoccupations en termes de bien-être. Ils 
affectent le système locomoteur des poulets et conduisent à des boiteries, dont la sévérité peut être mesurée par examen de la démarche des poulets. Les anomalies de la démarche sont probablement associées à de la douleur chez la plupart des oiseaux et, quoi qu'il en soit, peuvent affecter la capacité d'accès aux ressources (mangeoires, abreuvoirs) chez les individus les plus sévèrement affectés. Les anomalies de la démarche sont associées aux vitesses de croissance rapides. Toutefois, les résultats obtenus varient selon les études à cause de la diversité des génotypes, des modes de gestion de l'élevage, de l'âge des animaux auquel les mesures sont effectuées et des systèmes de notation de la démarche utilisés. Les scores de démarche auraient une faible héritabilité, mais plusieurs facteurs contribuant à la démarche tels que les varus-valgus et la dyschondroplasie tibiale sont hautement héritables et ont déjà été pris en compte dans certains schémas de sélection.

Le rapport recommande que la diminution des niveaux de boiterie sévère devienne une priorité et mentionne que cela doit se réaliser notamment en augmentant la pression de sélection sur ce facteur. Il recommande également que les poulets avec des boiteries sévères (scores 4 et 5 du système de notation de Bristol, Kestin et al 1992) soient systématiquement euthanasiés. Les sélectionneurs devraient être encouragés à rechercher des critères de sélection et à les utiliser pour diminuer efficacement ces boiteries.

\section{2 / Dermatites de contact}

Les dermatites de contact sont un problème de bien-être important. Elles se caractérisent par une coloration de la peau et une érosion des tissus. Dans les cas sévères, l'érosion des tissus aboutit à des ulcérations avec des réactions inflammatoires des tissus sous-cutanés. De telles lésions peuvent générer de la douleur. Elles sont largement répandues dans les élevages en Europe, avec des incidences variables selon les études et les pays. Les prévalences peuvent être très fortes dans certains cas. Leur étiologie est multifactorielle, impliquant des conditions environnementales (comme l'humidité de la litière par exemple) et des prédispositions génétiques. Plusieurs études réalisées sur des lots en conditions commerciales suggèrent, en effet, que le génotype peut influencer leur prévalence. Ces dermatites ont un degré modéré d'héritabilité.

Dans le rapport, il est recommandé que ce caractère soit inclus dans les programmes de sélection et qu'un système de notation harmonisé soit développé en Europe. Dans la continuité de cette recommandation, la Commission européenne est en train de réfléchir à une harmonisation de la notation en se basant sur les systèmes existants. Les filières sont fortement incitées à diminuer la proportion de poulets avec des dermatites de contact au cours des 10 prochaines années.

\section{3 / Ascite, syndrome de mort subite}

L'ascite, qui se caractérise par une accumulation de liquide dans la paroi abdominale, et le syndrome de mort subite sont des maladies d'origine métabolique. Elles sont létales. Il existe une prédisposition génétique pour ces deux maladies, ainsi qu'un lien entre la vitesse de croissance et l'ascite, et probablement aussi le syndrome de mort subite. Les génotypes à croissance lente sont plus résistants à l'ascite et une prise alimentaire faible en énergie, qui diminue la vitesse de croissance, peut diminuer le syndrome de mort subite. Les sélectionneurs prenant en compte la résistance à l'ascite depuis déjà plusieurs années, la prévalence de ce trouble a probablement diminué, mais cette tendance reste à confirmer en l'absence de données validées en élevage.

Le rapport recommande que la sélection contre ces deux affections se poursuive. Leurs prévalences ont besoin d'être évaluées systématiquement afin de s'assurer qu'elles restent faibles.

\section{4 / Mobilité}

La sélection pour une croissance rapide a eu un impact sur le comportement des poulets. Elle a entraîné une diminution de leur mobilité et une réduction de l'utilisation de leur environnement. Les résultats expérimentaux ne permettent pas de conclure si la faible mobilité est liée à un manque de motivation ou à une incapacité physique, même si certaines études suggèrent que ces deux aspects sont impliqués. Certains facteurs environnementaux tels que la lumière, la richesse de l'environnement et la densité d'élevage affectent eux aussi la mobilité des poulets.

Dans le rapport, il est recommandé de sélectionner pour une augmentation de la motivation des poulets à être actifs et notamment à se déplacer, en sélectionnant par exemple moins fortement sur la vitesse de croissance. Il est indiqué que les modes de gestion de l'élevage qui favorisent la mobilité des poulets doivent être encouragés.

\section{5 / Confort thermique}

Les génotypes à croissance rapide actuels sont moins tolérants aux stress de chaleur. Quelques études montrent que la résistance au stress de chaleur, évaluée par le maintien du gain de poids, de la température corporelle ou de la mortalité à des niveaux habituels lorsque la température est élevée, varie selon les génotypes. De plus, les risques d'inconfort thermique augmentent avec l'âge des animaux et la densité d'élevage, notamment à cause de l'augmentation de la production de chaleur par les animaux et de la baisse d'espace disponible. Ces facteurs limitent les possibilités de dissipation de la chaleur et favorisent l'augmentation de la température de la litière.

Dans le rapport, il est recommandé d'adapter les techniques de gestion de l'élevage pour éviter les stress thermiques, par exemple en réévaluant les températures ambiantes recommandées pour ces génotypes, car elles pourraient être trop élevées. Il est également recommandé de choisir des génotypes adaptés aux températures d'élevage, en particulier pour les régions chaudes.

\section{6 / Fonction digestive}

Dans le rapport, il est indiqué que les problèmes digestifs ont augmenté dernièrement et que cette évolution pourrait être liée à différents facteurs environnementaux (comme la composition des aliments) ou à des changements dans l'efficacité digestive des génotypes actuels et dans leur sensibilité aux maladies. Ces problèmes digestifs pourraient avoir des effets négatifs sur la qualité des litières (humidité) et favoriser le développement des dermatites de contact.

\section{7 / Sélection génétique et inter- action avec l'environnement}

L'amélioration globale du bien-être ou de certains caractères liés au bienêtre, au cours des dernières années, n'a pu être quantifiée du fait du manque d'accès aux données confidentielles des sélectionneurs. Dans le rapport, il est recommandé de mettre en place une évaluation standardisée du bien-être en élevage commercial qui soit harmonisée entre les différents pays européens, afin d'évaluer le niveau de ces différents caractères phénotypiques, ainsi que l'impact de la sélection génétique sur ces derniers.

Il existe de nombreuses et fortes interactions entre l'environnement et les caractéristiques génétiques des oiseaux en termes de bien-être. Ce n'est pourtant que récemment que les études sur la sélection génétique et son interaction avec l'environnement incluent des caractères de bien-être (uniquement des pathologies) en plus des caractères de 
production. Dans la conclusion du rapport, il est indiqué que le bien-être des poulets serait amélioré si les génotypes étaient sélectionnés en fonction de l'environnement d'élevage. En pratique, les schémas de sélection avicole ne s'y prêtent pas bien car les lignées en sélection sont le plus souvent élevées dans des conditions très contrôlées (en particulier au niveau sanitaire) qui peuvent être très éloignées des conditions de production du poulet de chair. La prise en compte des interactions entre génétique et environnement peut passer par l'élevage de cheptels de reproducteurs (ou de leurs collatéraux) dans des environnements contrastés (correspondant par exemple à des pays plus ou moins tempérés ou avec des qualités d'aliments variés). Les nouvelles possibilités ouvertes par la sélection génomique pourront également être mises à profit pour améliorer la capacité d'adaptation au milieu grâce à la recherche de marqueurs génétiques associés aux phénotypes mesurés sur les poulets de chair en production et utilisés en sélection pour le choix des reproducteurs.

Afin de garantir aux poulets de chair un bon niveau de bien-être, le rapport émet différentes recommandations : inclure les caractères de bien-être héritables dans les schémas de sélection, maintenir une diversité génétique pour s'adapter aux besoins futurs du marché et pouvoir développer des lignées qui puissent résister dans des environnements peu favorables, sélectionner et utiliser des lignées adaptées aux environnement locaux (par exemple par l'utilisation de génotypes à croissance plus lente pour les régions chaudes) ou à différents types d'environnements. Il est aussi recommandé de mettre en place un système d'évaluation du bien-être indépendant des sélectionneurs qui permette aux éleveurs de choisir en connaissance de cause le génotype le plus adapté à leurs propres conditions d'élevage. Les caractères liés au bien-être étant souvent difficiles à mesurer, leur introduction dans le cadre des schémas de sélection classiques pose des problèmes de faisabilité et de fiabilité des mesures. La sélection génomique pourrait alors être un outil utile pour améliorer les caractères de bien-être en permettant de sélectionner les animaux sans avoir à réaliser de mesures phénotypiques. La faisabilité d'une telle approche n'est pour l'instant pas démontrée mais les caractères de comportement sont aujourd'hui pris en compte dans plusieurs programmes de détection de Quantitative Trait Loci (QTL) (en France et à l'étranger) pour identifier les régions chromosomiques impliquées et les liens éventuels avec les caractères de production.

\section{2 / Reproducteurs paren- taux et grand-parentaux}

Le rapport de 2000 sur le bien-être des poulets élevés pour la production de viande (SCAHAW 2000) relève que les préoccupations majeures sont les désordres métaboliques qui génèrent, en particulier, des troubles au niveau des pattes, de l'ascite et le syndrome de mort subite. Il précise que des problèmes de bien-être apparaissent également à cause des conditions d'élevage des reproducteurs, notamment si l'on considère les restrictions alimentaires et d'espace.

La présente synthèse ne vise pas à résumer l'intégralité du rapport de l'EFSA sur les aspects relatifs aux problèmes de bien-être lié aux conditions d'élevage et de logement des reproducteurs, mais uniquement à présenter ceux en lien avec la sélection génétique. Au cours des 30 dernières années, le potentiel de croissance des individus a, en effet, fortement augmenté. Ceci a eu plusieurs impacts au niveau du bien-être des reproducteurs :

- le recours à une restriction alimentaire importante, surtout pendant la période d'élevage, de façon à ne pas avoir d'animaux en surpoids et à garantir de bonnes performances de reproduction,

- une agressivité entre les animaux liée, entre autres, à la forte restriction alimentaire,

- des problèmes locomoteurs en cas de surpoids,

- des troubles métaboliques.

De plus, certaines pratiques de sélection, comme l'utilisation des cages individuelles conventionnelles, sont aujourd'hui remises en question.

\section{1 / Restriction alimentaire}

La sélection génétique pour une plus forte croissance dès le jeune âge des animaux conduit à des prises alimentaires trop importantes si les reproducteurs sont nourris ad libitum pendant de longues périodes. L'absence de restriction alimentaire entraîne généralement un surpoids associé à différents problèmes de bien-être comme les boiteries et la mort subite, ainsi qu'une diminution de la fertilité. Une étude a notamment montré des taux de mortalité de $40 \%$ à 40 ou 49 semaines d'âge quand les poulets étaient nourris ad libitum contre $6 \%$ quand ils étaient restreints. D'un autre côté, la restriction alimentaire induit une sensation de faim, de la compétition pour accéder aux mangeoires et de l'agressivité pouvant conduire à des blessures graves.

La restriction alimentaire commence généralement dès une semaine d'âge et les rations sont restreintes au tiers ou au quart de la ration ingérée spontanément par l'animal. La prise alimentaire journalière se fait rapidement, généralement en moins de 15 minutes. Il existe aussi des programmes alimentaires dans lesquels les animaux ne sont pas alimentés un ou deux jours par semaine. Les conséquences sur le bien-être des animaux sont la faim chronique, la réalisation d'activités anormales comme la prise de boisson trop importante, le picage massif de supports non alimentaires et les déambulations. La restriction alimentaire en période de ponte est bien moins sévère avec, jusqu'au pic de ponte, une ration allant de 45 à $80 \%$ de la ration ingérée spontanément. Après le pic de ponte, ce pourcentage atteint $80 \%$ de la ration prise ad libitum. En période de ponte, bien que les effets de la restriction alimentaire soient moins sévères qu'en période d'élevage (avant la ponte), on peut tout de même observer plus de picage d'objets et de prise de boisson que chez les animaux non restreints.

En vue de réduire les effets négatifs de la restriction alimentaire, plusieurs solutions ont été envisagées. La dilution de la ration et l'utilisation de suppresseurs d'appétit ont ainsi été testées, mais leur efficacité n'a cependant pas été clairement établie. Dans certains pays européens comme la France, l'utilisation de souches à croissante lente (type Label Rouge) ou intermédiaires permet d'appliquer aux reproducteurs une restriction alimentaire moins forte. C'est également le cas pour les femelles reproductrices naines dites «mini» utilisées pour la production de poulets standard, à croissance lente ou intermédiaire. Cette génétique particulière permet d'appliquer un rationnement alimentaire moins sévère, d'augmenter la durée de prise alimentaire (quelques heures) et de diminuer les chargements (poids par mètre carré). Cette production représente $98 \%$ des stocks de parentaux français et 18 à $20 \%$ en Europe.

Pour réduire ce problème de restriction alimentaire, le rapport recommande la sélection de génotypes qui requièrent une moindre restriction alimentaire, ce qui pourrait impliquer une réduction de leur vitesse de croissance. L'évolution dans le temps du niveau de restriction alimentaire appliqué aux reproducteurs devrait être surveillée. Le rapport recommande également d'évaluer l'impact sur le bien-être de stratégies alimentaires alternatives. 


\section{2 / Agression et mutilation}

Pendant la période d'élevage, la restriction alimentaire engendre de la compétition au moment des repas. Une augmentation du nombre d'agressions est observée chez les animaux restreints par rapport aux animaux non restreints. Elle est le fait des mâles et des femelles et intervient aux abords des mangeoires. Par ailleurs, il est très important de veiller à ce que les mâles n'atteignent pas leur maturité sexuelle avant les femelles ce qui pourrait conduire à des agressions de la part des mâles et à une augmentation du nombre de blessures chez les femelles. Ces dernières peuvent également fuir ou se dissimuler dans les nids pour éviter la copulation. Dans le rapport, il est recommandé d'utiliser des techniques de distribution d'aliment diminuant la compétition alimentaire et, ainsi, les blessures. Un outil de mesure de la qualité du plumage (surface dénudée) et des lésions devrait être développé afin de mesurer l'impact des comportements agressifs sur les animaux.

Les mutilations sont réalisées principalement sur les mâles pour limiter les conséquences des agressions des mâles entre eux ou envers les femelles (débecage, coupe de la crête et des phalanges) ou des blessures liées à la copulation (ablation des ergots). Les mutilations, pratiquées sans anesthésie, induisent très vraisemblablement de la douleur, mais l'absence de mutilation peut conduire à du picage, du cannibalisme ou des blessures graves d'autre nature. Il est recommandé de réaliser des enquêtes sur le taux de réalisation de ces mutilations et sur leur réelle utilité au cas par cas.

\section{3 / Troubles locomoteurs et métaboliques}

Les problèmes locomoteurs des reproducteurs sont de même nature que ceux du poulet de chair, néanmoins ils sont moins fréquents en raison des restrictions alimentaires. En revanche, en l'absence de restriction, les taux d'euthanasie des mâles pour cause de problèmes locomoteurs sont élevés et la rupture de tendon peut toucher environ $50 \%$ des femelles des troupeaux entre 23 et 35 semaines de ponte. Les problèmes métaboliques comme le syndrome de mort subite peuvent être observés chez les femelles et seraient probablement dus à des hypocalcémies et des hypokaliémies.

Le rapport souligne le manque de données disponibles sur l'évolution des troubles locomoteurs sur les vingt dernières années. En conséquence, un système de notation devrait être développé pour suivre les tendances dans le temps.
Il faudrait aussi évaluer les relations entre boiteries et douleurs.

\section{4 / Elevage en cage}

Une petite proportion des parentaux et grand-parentaux en Europe est élevée en cage avec une reproduction par insémination artificielle. Bien qu'il n'y ait pas de littérature sur l'effet de ces cages conventionnelles sur le bienêtre des reproducteurs, on peut penser que les problèmes sont les mêmes que pour les poules pondeuses avec une absence de litière, de perchoirs et de nids, ainsi qu'une surface disponible restreinte. Ces cages ne peuvent donc satisfaire les besoins comportementaux des animaux et il est recommandé, dans le rapport, qu'elles soient aménagées comme pour les poules pondeuses.

\section{3 / Besoins de recherche}

Comme dans les autres rapports de l'EFSA, des recommandations sont faites sur les recherches qu'il apparaît nécessaire de développer.

Dans le cas des poulets de chair, il faudrait développer des recherches sur les interactions entre les facteurs environnementaux et génétiques dans le domaine du bien-être. Il faudrait également: 1/ Identifier les causes de la mobilité réduite des animaux et déterminer l'impact de cette mobilité réduite sur le bien-être, 2/ Développer des méthodes de surveillance du bien-être, de la santé et de la mortalité, de façon à évaluer objectivement et enregistrer le bien-être dans les lots commerciaux.

Dans le cas des reproducteurs, il est notamment recommandé de faire des investigations sur l'impact de la réduction de la vitesse de croissance sur le bien-être, la santé et les performances des reproducteurs (faim, frustration, désordres métaboliques...), ainsi que : 1) Etudier le comportement des reproducteurs en restriction alimentaire dans les lots commerciaux, 2/Déterminer le niveau approprié de restriction qui permet aux animaux de ne pas avoir faim tout en ne souffrant pas des problèmes de santé liés à une alimentation non restreinte, 3/ Etudier les caractères génétiques qui pourraient être utilisés pour réduire les comportements agressifs et la façon de réduire les blessures faites aux femelles pendant la copulation.

\section{Conclusion}

Face au manque de données issues du terrain, il apparaît nécessaire de déve- lopper des outils de mesures fiables du bien-être (incluant les causes de mortalité), afin de réaliser un suivi objectif des problèmes rencontrés à la fois chez les reproducteurs et les poulets de chair. Ce suivi devrait être réalisé de façon indépendante.

L'analyse des facteurs de risque associés aux problèmes de bien-être s'est avérée difficile à cause des interactions entre le génotype et l'environnement des animaux. Cependant, une composante génétique est mise en évidence pour plusieurs indicateurs de bien-être. C'est pourquoi le rapport sur les poulets de chair recommande d'augmenter la pression de sélection sur ces caractères héritables (dermatite de contact, ascite, syndrome de mort subite). Un critère de sélection pour la sensibilité aux boiteries, auxquelles de nombreux facteurs contribuent, devrait aussi être recherché pour empêcher le développement de boiteries sévères. Par ailleurs, une limitation de la vitesse de croissance pourrait permettre de favoriser la capacité ou la motivation des poulets à se déplacer ou, encore, le confort thermique des animaux. D'autres stratégies de sélection qui favorisent l'adaptabilité à différents environnements et qui intègrent des caractères de bien-être sont aussi à envisager. Chez les reproducteurs, le rapport a fait ressortir le rôle central de la restriction alimentaire qui génère de la faim et entraîne des agressions. Les principaux leviers d'action sont la réduction de la vitesse de croissance et le développement de nouvelles conduites d'alimentation. Par ailleurs, le rapport sur les reproducteurs précise que les conditions de logement des reproducteurs devraient s'aligner sur les minimums requis chez les poules pondeuses dans le cas de l'élevage en cage.

Les suites données à ces rapports par la Commission européenne ne sont pas encore connues. Cependant, les réflexions issues de ces expertises mettent en évidence la nécessité d'une plus grande intégration des caractères de bien-être dans les schémas de sélection et de l'évaluation de leurs impacts dans les conditions de production. Si les objectifs de sélection en filière chair visent à améliorer les performances des croisements commerciaux, ils ont aussi des conséquences sur les reproducteurs. L'évaluation des conséquences des choix de sélection sur le bien-être devrait donc intégrer les différents maillons de la sélection.

A l'issue de ces expertises, il paraît d'actualité d'envisager une baisse de la pression de sélection sur la vitesse 
de croissance. Cette évolution des pratiques pourrait relever soit d'une démarche volontaire, soit d'une contrainte imposée par le législateur. Quoiqu'il en soit, les pertes économiques inhérentes à une telle évolution pourraient s'avérer être compen- sées, au moins en partie, par les gains générés par une meilleure santé des animaux, une diminution de la mortalité, voire même une meilleure image de la production auprès des citoyens et des consommateurs.

\section{Remerciements}

Nous remercions les experts et les membres de l'EFSA ayant contribué à l'élaboration des deux rapports.

\title{
Références
}

AHAW, 2010a. Scientific Opinion on the influence of genetic parameters on the welfare and the resistance to stress of commercial broilers. EFSA J., 8, 1666. 82p. http:/ /www.efsa.europa.eu

AHAW, 2010b. Scientific Opinion on welfare aspects of the management and housing of the grand-parent and parent stocks raised and kept for breeding purposes. EFSA J., 8, 1667. 81p. http://www.efsa.europa.eu

Arnould C., 2003. Le bien-être des poulets de chair : problèmes et solutions. In :
Hors-série, Bien-être : la nouvelle donne en aviculture et cuniculture. Sciences et Techniques Avicoles, Septembre, 17-21.

Arnould C., Leterrier C., 2007. Bien-être animal en élevage de poulets de chair. In : Numéro spécial, Bien-être animal. INRA Prod. Anim., 20, 41-46.

Beaumont C., Le Bihan-Duval E., Juin H., Magdelaine P., 2004. Productivité et qualité du poulet de chair. INRA Prod. Anim., 17, 265273.
Kestin S.C., Knowles T.G., Tinch A.E., Gregory N.G., 1992. Prevalence of leg weakness in broiler chickens and its relationship with genotype. Vet. Rec., 131, 190-194.

SCAHAW, 2000. The welfare of chickens kept for meat production (broilers). Report of the scientific committee on animal health and animal welfare (adopted 21 March 2000), European commission, Health and consumer protection directorate-general. 149p. http:/ leuropa.eu.int/comm/food/fs/sc/scah/outcome en.html

\section{Résumé}

En prolongement des conclusions du rapport de la Commission européenne de 2000 sur le bien-être du poulet de chair et dans le cadre de la directive 2007/43/CE, la Commission européenne a confié à l'European Food Safety Authority l'élaboration de deux avis scientifiques. L'un concerne l'impact de la génétique sur le bien-être et la résistance au stress des poulets de chair, l'autre l'impact de la gestion de l'élevage et du logement sur le bien-être des reproducteurs grand-parentaux et parentaux. Cette synthèse s'appuie sur le contenu de ces deux avis publiés en 2010, suite à une expertise scientifique réalisée en 2009/2010. Elle précise tout d'abord en quoi les principaux problèmes de bien-être rencontrés chez les poulets de chair (troubles locomoteurs, dermatites de contact, ascites, syndromes de mort subite, mobilité limitée, confort thermique...) peuvent être affectés par la sélection génétique sur une croissance rapide et un indice de consommation faible. La question des interactions génotype-environnement est également abordée. Sont exposés ensuite les principaux problèmes de bien-être rencontrés chez les reproducteurs en se focalisant sur ceux ayant un lien avec la sélection sur la croissance (restriction alimentaire, agressions entre congénères, mutilations...). Enfin, sont présentés les besoins de recherche qui ont été mis en évidence lors de la rédaction de ces avis et les principales conclusions.

\begin{abstract}
Genetic and welfare in commercial broiler chickens and breeders

Conclusions from the report of the European Commission of 2000 on «the welfare of chickens kept for meat production (broilers) » and the directive 2007/43/EC led the European Commission to request the European Food Safety Authority to issue two scientific opinions, the first one on the influence of genetic parameters on the welfare and resistance to stress of commercial broilers and the second one on the welfare of grand-parent and parent stocks raised and kept for breeding purposes. The present synthesis is based on these two opinions published in the EFSA Journal in 2010 and which came after a scientific expertise conducted in 2009 / 2010. This synthesis will first state how the main welfare problems encountered in broiler chickens (leg problems, contact dermatitis, ascites, sudden death syndrome, reduced mobility, thermal discomfort...) can be exacerbated by intense selection for fast growth rate and increased feed efficiency. Second, the main welfare problems encountered in breeders will be stated focusing on those that have links with selection on fast growth rate (feed restriction, aggression, mutilations...). Then, the need for future research and main conclusions will be presented.
\end{abstract}

ARNOULD C., MICHEL V., LE BIHAN-DUVAL E., 2011. Sélection génétique et bien-être des poulets de chair et des reproducteurs. In : Bien-être du poulet de chair. Dossier, INRA Prod. Anim., 24, 165-170. 